\title{
Matiz feminizante del tuteo y el futuro del voseo en el departamento de Antioquia (Colombia)
}

\author{
The feminizing nuance of tuteo and the future of voseo in the department of \\ Antioquia (Colombia)
}

\author{
Ji Son Jang \\ Universidad de Antioquia, Facultad de Comunicaciones, Medellín, Colombia. \\ Correo electrónico: tetsuji_miyahara@hotmail.com
}

En el presente artículo se muestran algunos aspectos sobre el tuteo y el voseo en el departamento colombiano de Antioquia desde una perspectiva sociolingüística, con base en los datos obtenidos principalmente a través de las encuestas aplicadas a informantes en dicha zona. De la literatura existente sobre el tema y del resultado del análisis de los datos se puede afirmar que el tuteo, en comparación con el voseo, se considera como un tratamiento nuevo y paulatinamente se está expandiendo en esta zona. No obstante, sería necesario un largo periodo de tiempo para que el tuteo alcanzara el nivel de voseo, debido a su matiz feminizante y al hecho de que el voseo forma parte de la identidad regional de los antioqueños.

Palabras clave: tuteo, voseo, Antioquia (Colombia), matiz feminizante, identidad

In this paper it is that are shown some aspects of the tuteo and the voseo in the Colombian department of Antioquia from the sociolinguistic perspective, based primarily on data obtained through surveys of informants in the area. In the existing literature on the subject and the result of data analysis, it can be said that the tuteo, compared the voseo, is considered as a new address form in this department and is gradually expanding in this area. Nevertheless, it will be long before the tuteo becomes as frequent as the voseo, as the former is traditionally perceived as feminizing, and because the voseo is ingrained in the regional identity of people from Antioquia.

Key words: tuteo, voseo, Antioquia (Colombia), feminizing nuance, identity

\section{INTRODUCCIÓN}

De acuerdo con Brown y Levinson, la selección pronominal se considera como una de las estrategias de cortesía fundamentales en la interacción verbal (1987: 107, 179-181 ${ }^{1}$. Asimismo, varios estudiosos han señalado la importancia de adquirir

\footnotetext{
El tú y el vos se consideran como una de las estrategias de cortesía positiva o de solidaridad, y el usted como una de
} 
un buen manejo de los pronombres personales mediante los cuales se transmite directamente el comportamiento lingüístico cortés o descortés (Braun 1988: 46; Bravo 2001: 304; Iglesias 2001: 256; Boretti y Rigatuso 2004: 145; Guerra 2008: 228; Placencia 2010: 344). Por un uso pronominal inadecuado puede generarse una situación conflictiva hasta entre interlocutores nativos de la misma lengua (Páez 1981: 49; Bolívar 2001: 68; Blas 2005: 19; Hummel 2010a: 109), y desde esta óptica se han realizado numerosos estudios acerca de las fórmulas de tratamiento en el mundo hispanohablante (Blas 1994-1995, 1995, 2003; Serrano 2000: 204; Móccero 2003: 346; Carrera y Álvarez 2004; Navarro 2004; Medina 2010).

Las normas en el uso de las fórmulas de tratamiento en el mundo hispanohablante se han ido transformando a lo largo del tiempo, por factores geográficos, políticos y/o socioeconómicos en cada región (Carrera y Álvarez 2004: 227; Navarro 2004: 216). No obstante, en el departamento de Antioquia - considerado como centro del voseo $^{2}$ en Colombia-, donde coexisten tres formas pronominales: tú, vos y usted, tras la publicación del trabajo de Montes (1967) no se habían realizado suficientes investigaciones de carácter empírico en las que se incluyeran datos actualizados sobre el empleo de estas formas de tratamiento. Con el ánimo de llenar esta laguna, hemos comenzado a indagar este fenómeno en distintos tipos de interacción en la ciudad de Medellín con un enfoque sociopragmático entre la población joven universitaria, tomando como variables el estrato socio-económico ${ }^{3}$, el sexo (Jang 2010), el origen urbano o rural de los informantes y sus padres, y la edad media de los informantes (Jang 2012).

Desde una perspectiva diacrónica amplia, con base en los estudios de Montes (1967) y Páez (1981), entre otros, cabe señalar que el tú en el departamento de Antioquia se considera más bien como un trato no propio de esta región, o lo que es lo mismo, es un trato nuevo comparado con el vos. Teniendo en cuenta esto y que, por lo general, el cambio de la norma pronominal se ha generado en zonas urbanas y no en zonas rurales (Álvarez y Carrera 2006: 123; Fontanella 1970: 17, 1985: 21; Molina 2002: 114; Kluge 2005: 187), en el estudio de Jang (2012) sobre la selección pronominal en la ciudad de Medellín, se partió de la hipótesis de que en la mayoría de los casos interpersonales los informantes de origen urbano cuyos padres son del mismo origen emplearían el tú más frecuentemente que el vos, en comparación con los informantes de origen rural cuyos padres son del mismo origen. Sin embargo, esta hipótesis no se corroboró excepto en apenas algunos casos, especialmente en la relación novio/novia.

las estrategias de cortesía negativa o de distanciamiento. Varios estudios han señalado que, aunque haya diferencias regionales en el aspecto pragmático de los pronombres tú/vos y usted, por lo general, tú/vos se asocian con el concepto de solidaridad y usted con el de no-solidaridad (Pedroviejo 2004: 246; Serrano 2000: 204). A este respecto, vale la pena mencionar el trabajo de Brown y Gilman (1960), considerado como pionero, en el cual se menciona el concepto de poder y solidaridad para la selección pronominal.

2 El voseo moderno es el tratamiento familiar empleado para referirse a la segunda persona del singular. Existen algunas regiones hispanoamericanas donde el voseo se emplea a través del pronombre tú con el voseo verbal, por ejemplo, en la capital uruguaya (J. Steffen 2010: 454) o la chilena (Uber 2010: 1053). El voseo medellinense, no obstante, siempre se realiza a través del pronombre vos con el voseo verbal (Jang 2009).

3 En la sociedad colombiana está oficialmente establecido el sistema de estratos socioeconómicos que se dividen en seis grados: uno es el más bajo y seis es el más alto. 
En este artículo se presenta una parte de los resultados acerca del uso de tú derivados de una investigación sobre la selección pronominal llevada a cabo en tres municipios antioqueños: 1) ciudad de Medellín ${ }^{4}$, que se considera zona urbana; 2) Carmen de Viboral, que se entiende como una zona semirural situada a una hora de la ciudad en automóvil; y 3) Andes, que se entiende como una zona rural y se sitúa a unas tres horas de la ciudad en automóvil, con el fin de observar principalmente si el tuteo es predominante en la zona urbana en comparación con la zona rural. La población estudiada está constituida por informantes universitarios de edades comprendidas entre los 13 y los 34 años. La variable social para esta investigación es el lugar de residencia de los informantes, es decir, zona urbana (Medellín), semirural (Carmen de Viboral) o rural (Andes). En cuanto al origen de la población estudiada, se trabajó solamente con informantes de origen antioqueño cuyos padres también son del departamento de Antioquia, con el fin de evitar cualquier influencia de usos pronominales de otras regiones, las cuales se encuentran fuera del objetivo de este trabajo.

\section{CORPuS}

El corpus principal del presente estudio se obtuvo a través de encuestas aplicadas a informantes universitarios en las tres zonas mencionadas ${ }^{5}$. Dado que la única variable social analizada es el lugar de residencia de los informantes (zona urbana, semirural y rural), y también debido a la dificultad para conseguir a informantes de los estratos altos en las zonas semirural y rural, hemos decidido trabajar únicamente con informantes de los estratos populares, es decir, 1, 2 y $3^{6}$.

La gran mayoría de los cuestionarios se aplicaron en instituciones universitarias o en colegios en dichas áreas. En el cuestionario se preguntó a los informantes con qué pronombre (tú, vos o usted) tratarían a cada interlocutor. Los interlocutores que se presentan en el cuestionario se diseñaron teniendo en cuenta los conceptos de poder relativo y de distancia presentados por Brown y Levinson (1987: 76) en la ecuación $\mathrm{W}=\mathrm{D}(\mathrm{S}, \mathrm{H})+\mathrm{P}(\mathrm{H}, \mathrm{S})+\mathrm{R}^{7}$.

En concreto, los interlocutores son: 1$)^{8}$ su madre; 2) su padre; 3) su hermana mayor; 4) su hermana menor; 5) su hermano mayor; 6) su hermano menor; 7) su abuela; 8) su abuelo; 9) su hija de 20 años; 10) su hija de 5 años; 11) su hijo de 20 años; 12) su hijo de 5 años; 13) su novio/novia (en privado); 14) su novio/novia (ante sus amigos); 15) su novio/novia (ante sus padres); 16) su novio/novia (ante desconocidos); 17) su esposo/esposa o compañero/compañera; 18) una amiga 10 años mayor; 19) una amiga de su misma edad; 20) un amigo 10 años mayor; 21) un amigo de su misma edad; 22) una señora de 60 años que no conoce; 23) una

\footnotetext{
Capital del departamento de Antioquia, incluyendo su área metropolitana.

5 Los datos de la presente investigación se recogieron entre septiembre de 2010 y marzo de 2011.

6 Para el análisis sobre la selección pronominal en Medellín con las variables estrato socioeconómico y sexo, véase Jang (2010); con las variables origen urbano / rural antioqueño, véase Jang (2012).

7 W (weightiness) corresponde al peso del FTA (face threatening act). D (distance) es la distancia social que media entre S (speaker, a saber, hablante) y H (hearer, es decir, oyente). P (power) representa el poder que el oyente tiene sobre el hablante. R (rank) se refiere al grado de imposición del acto de habla.

8 Estos numerales corresponden a los números de las preguntas del cuestionario.
} 
joven de 20 años que no conoce; 24) un señor de 60 años que no conoce; 25) un joven de 20 años que no conoce; 26) una niña de 5 años; 27) un niño de cinco años; 28) su profesora de 60 años muy amiga; 29) su profesora de 30 años muy amiga; 30) su profesor de 60 años muy amigo; 31) su profesor de 30 años muy amigo; 32) su profesora de 60 años con la que no tiene confianza; 33) su profesora de 30 años con la que no tiene confianza; 34 ) su profesor de 60 años con el que no tiene confianza; 35) su profesor de 30 años con el que no tiene confianza; 36) una médica de 60 años en su consultorio; 37) una médica de 30 años en su consultorio; 38) un médico de 60 años en su consultorio; 39) un médico de 30 años en su consultorio; 40) un sacerdote de 60 años; 41) un sacerdote de 30 años.

Por otro lado, con el fin de buscar alguna relación entre la preferencia del tú o del vos y la evaluación pronominal, en el cuestionario se formuló la siguiente pregunta: ¿Usted considera que el uso del tú (por ejemplo, “tú eres”) suena más refinado que el uso del vos (por ejemplo, "vos sos”)? Sí: _ No: _ Por último, con el propósito de reconfirmar el matiz feminizante del tuteo (Jang 2009, 2010) se realizó la siguiente pregunta: ¿Usted considera que el uso del tú (por ejemplo, "tú eres”) entre hombre y hombre puede sonar feminizante (afeminado)? Sí: No:

\section{BREVE DESCRIPCIÓN HISTÓRICA DEL VOSEO FRENTE AL TUTEO}

\subsection{En la Península Ibérica}

La norma del tratamiento tú y vos en la Península Ibérica dependía del grado de igualdad en la sociedad de cada época. A medida que la sociedad ibérica iba cambiando, el valor del tratamiento también fue modificándose (Páez 1981). De acuerdo con Páez (1981: 39-44), en los siglos XIII y XIV vos tenía dos valores: a) vos reverencial que se otorgaba en razón del rango estamental superior, y b) vos de distanciamiento social que se otorgaba a individuos de estratos inferiores. Según Páez (1981: 45-54), esta doble dimensión de vos generó un problema serio, ya que siendo indicador de rango estamental superior igualmente se utilizaba como indicador de estratos inferiores. Esto es, cuando se utilizaba vos desde las personas inferiores a las superiores no se sabía claramente si este tratamiento se empleaba con valor reverencial o despectivo. Por esta razón, surgió la necesidad de crear otro tratamiento que indicara claramente reverencia. De esta manera, con el tiempo, vos adquirió un valor despectivo y generó una nueva forma de tratamiento de reverencia, vuestra merced $^{9}$, que posteriormente se transformaría en $u_{s t e} d^{10}$ para evitar la significación de inferioridad social que conllevaba vos. Con el transcurso del tiempo, vos desapareció en el habla cotidiana hispánica.

\subsection{En América}

Entre los peninsulares recién llegados al Nuevo Mundo a finales del siglo XV no existía una diferencia estamental tan marcada. Esa sensación de relativa igualdad hizo

\footnotetext{
9 Entre otros, véase Moreno (1986: 88).

10 Véase, por ejemplo, Álvarez y Carrera (2006: 123).
} 
que se generalizara el empleo del mismo pronombre vos, puesto que tú no se usaba tanto como vos ya que tenía cierto matiz despectivo, y aunque tenía una función de tratamiento de solidaridad, ésta fue asumida por vos. Asimismo, al comienzo no se generalizó el empleo de vuestra merced, ya que cuando los primeros peninsulares se establecieron en América, apenas comenzaba a utilizarse dicho tratamiento en la Península Ibérica, de modo que para los conquistadores o colonizadores no era una forma familiar. En cambio, para ellos vos ya era una forma bien arraigada que aún mantenía un sentido respetuoso, a pesar de que en la Península Ibérica se hubiera ido perdiendo el prestigio de este pronombre y desapareciera del todo más adelante (Páez 1981: 61-70). Así, de la misma manera en que se ha observado en la historia peninsular, América también ha experimentado varios cambios sociales que, a su vez, han motivado cambios en lo lingüístico y, por supuesto, en el sistema pronominal.

A finales del siglo XV, la época en que los peninsulares se trasladaron al Nuevo Mundo, la política peninsular exhortaba a la intensificación del uso de vuestra merced y tú, y a la desvaloración de vos en América debido a la doble dimensión problemática del voseo ya mencionada. De esta manera, el voseo desapareció en algunas regiones hispanohablantes y el tuteo fue ganando terreno. Algunos estudiosos relacionan la desaparición o la permanencia del voseo, así como su grado de vitalidad, con factores geográficos, más concretamente, con el nivel de intensidad de los lazos con la política o la cultura de la Península Ibérica (Cisneros 1996: 40; Fontanella 1976: 269; Montes 1967: 22-23). Las regiones hispanoamericanas que tenían fácil acceso a la política o a la cultura peninsular, como Ciudad de México o Lima donde había virreinatos (Páez 1981: 76, 98) -o sus alrededores- (Hummel 2010b: 295-296), siguieron el cambio lingüístico que se estaba imponiendo, mientras que donde no existía esta facilidad, no se siguió este cambio.

A modo de ejemplo, el voseo desapareció en el español del altiplano central mexicano, donde había un virreinato y donde ejercía una fuerte influencia la política peninsular de la época (Vázquez y Orozco 2010: 249-252). Sin embargo, aún se observan formas voseantes en algunas regiones del sur de México (Vázquez y Orozco 2010: 256), lo cual implica que la política peninsular que exhortaba el tuteo en lugar del voseo no llegó suficientemente hasta zonas alejadas del centro político mexicano. Por su parte, Hummel (2010b: 295-296) afirma que en México, Perú, Santo Domingo y también en $\mathrm{Cuba}^{11}$, que fueron centros políticos donde existía un contacto fuerte con la Península Ibérica, se expandió rápidamente el tuteo. En lo que se refiere a las Antillas, este mismo estudioso señala que: "La victoria del tuteo sobre el voseo en las Antillas se explica unánimemente por la fuerte y larga vinculación del grupo isleño con España desde los primeros tiempos de la colonización" (Hummel 2010b: 295). En lo que atañe a Perú, Hummel (2010c: 395) indica:

Se confirma la teoría según la cual la extensión del tuteo frente al voseo se encuentra en correlación positiva con el influjo de España en la época colonial y con la propagación de la norma culta a partir del siglo XIX. De acuerdo con ello, el tuteo exclusivo se observa primero en el habla culta de las grandes ciudades a partir de finales del siglo XVIII, extendiéndose desde estos centros a través del país, primero en las capas cultas de las aglomeraciones urbanas, acompañado de un proceso de difusión de arriba hacia abajo, al menos en

von Büchau (2006: 134) señala la falta de comunicación con el centro político como la razón por la cual en la ciudad de Camagüey, Cuba, sobrevivió el voseo. 
los estratos sociales que adoptan el habla de la gente ilustrada como modelo. El voseo, a su vez, quedó confinado en las periferias norte y sur del Perú con tendencias hacia la extinción completa, sobre todo en el norte.

De acuerdo con Torrejón (2010: 418, 421) el voseo en Chile pervivió debido a su condición geográfica aislada de los centros administrativos y culturales como México, Lima y Santo Domingo. Para el caso de la selección pronominal paraguaya, M. Steffen (2010: 434) señala:

En singular, como tratamiento deferencial se usa usted y la única forma empleada para el tratamiento de confianza es el vos (Nota 8: Como veremos en el § 2.1, algunos autores constatan el uso de tú en Paraguay.). El uso de esta forma de tratamiento, arcaica desde la perspectiva del español peninsular, se debe a que el Virreinato del Río de la Plata, al que pertenecía también Paraguay, fue fundado apenas en 1776, más de dos siglos más tarde que los virreinatos con sede en Lima (1542) y México (1535). Las regiones virreinales que fueron fundadas más tarde estuvieron marginadas de España durante mucho tiempo y no adoptaron los cambios lingüísticos que se daban allí.

\section{Relaciones entre el voseo/tuteo y las zonas rural/urbana. Connotación del VOSEO}

En algunas regiones hispanohablantes el tuteo predomina en las zonas urbanas y, por ende, puede calificarse positivamente; en cambio, el voseo prevalece en las zonas rurales y, por ello, puede recibir una evaluación negativa. De acuerdo con Vázquez y Orozco (2010: 257), en México el voseo tabasqueño es un rasgo lingüístico sin prestigio, evaluado como propio de hablantes rurales o analfabetos. En Perú, en las primeras décadas del siglo XX, a diferencia de los centros del país como Lima o Cuzco, el voseo subsistía vivamente en la parte norte y sur, especialmente en los estratos populares de la ciudad y en las áreas rurales de estas regiones, con un matiz arcaizante (Hummel 2010c: 377). De este modo, se considera que el voseo peruano es propio de la zona rural, especialmente de los estratos más bajos y, por consiguiente, connota un matiz provinciano (Hummel 2010c: 384-385). En Cuba el voseo connota falta de instrucción o incultura y se considera vulgar, incorrecto o campesino, ya que no pertenece a la norma culta (Hummel 2010b: 311). En Panamá el voseo suele observarse en las zonas rurales y en la gente mayor (Moser 2010: 282283). En Guatemala el tuteo no se observaría casi entre la clase baja, los indígenas o la población rural (Moser 2010: 278).

En Chile, en el siglo XIX, el tuteo como una norma culta iba a ganando terreno sobre el voseo que se consideraba como signo de incultura, aunque el voseo era mucho más vital que el tuteo (Torrejón 2010: 420-421). Durante el siglo XX el voseo chileno siguió siendo el tratamiento predominante en los sectores populares urbanos y entre campesinos, mientras que el tuteo era corriente en la clase media y alta (Torrejón 2010: 421-422) ${ }^{12}$. Hummel (2010a: 104), por su parte, señala que el tuteo en este país

\footnotetext{
Este autor indica que, paulatinamente y cada vez más, el voseo sigue ganando terreno sobre el tuteo, hasta entre los jóvenes de las clases media y alta, debido a la debilitación del control social impuesto sobre los usos lingüísticos (Torrejón 2010: 421). A nuestro juicio, este proceso es el que se produce después del hecho de que el tuteo gane terreno sobre el voseo por cuestión de, verbigracia, identidad (Jang 2013).
} 
se considera más culto que el voseo e implica que el tú es utilizado más bien por los habitantes de los estratos altos de las zonas urbanas. En la zona urbana boliviana tú conlleva un matiz culto, y vos, popular y coloquial (Hummel 2010d: 401-402) ${ }^{13}$.

\section{Matiz Feminizante del tuteo}

Se ha reportado que en algunas regiones hispanohablantes el uso de tú conlleva un matiz feminizante. El tuteo costarricense igualmente puede indicar afeminación. En Maracaibo (Venezuela) tú tiende a ser usado más por las mujeres jóvenes y, por ello, los hombres jóvenes prefieren no emplearlo o lo toman -según el contextocomo un rasgo de afeminamiento. En Argentina, a comienzos de la década de los ochenta del siglo XX, se observa que las mujeres usan el vos con formas verbales de tú (verbigracia, vos comes) con más frecuencia que los hombres (Páez 1981: 105). Esta última observación es bastante interesante: en Argentina no existe tú como pronombre de segunda persona singular de solidaridad de carácter no marcado, pero si existiera, muy posiblemente lo usarían más las mujeres jóvenes que los hombres jóvenes. Asimismo, en Medellín (Colombia), el tú puede sonar feminizante cuando se emplea entre interlocutores masculinos y, en consecuencia, esta forma de tratamiento es utilizada con más frecuencia por las mujeres (Jang 2010: 87).

\section{Tratamiento Estadístico de datos}

En esta investigación se apeló a tres técnicas estadísticas: 1) tamaño de muestra óptimo para distribuciones multinomiales (Thompson 1987); 2) análisis de varianza con un solo factor (ANOVA), y; 3) prueba de hipótesis para proporciones. Este procesamiento se ha realizado con el programa $R$.

\subsection{Tamaño de muestra óptimo para distribuciones multinomiales}

El tamaño de la muestra tiene como objetivo seleccionar aleatoriamente el menor número de observaciones $n$ que represente a la población total (Thompson 1987). Los tres parámetros definidos para la estimación del tamaño de la muestra fueron:

- Nivel de confianza del 99\% (1- $\alpha$ con $\alpha=0,01)$.

- Precisión absoluta de $+5 \%(d=0,05)$.

- $m=3$ (Las categorías para las fórmulas de tratamiento pronominales: tú, vos, usted).

\subsection{Análisis de varianza con un solo factor (ANOVA)}

Se apeló a la prueba del análisis de varianza con un solo factor (ANOVA) con el fin de verificar si la edad media en las tres zonas son iguales o no, es decir, para determinar la influencia de la edad en el análisis de los datos. Si la prueba nos muestra

De todas maneras, según este autor (Hummel 2010d: 410), de manera general Bolivia es un país claramente voseante, hasta en los estratos cultos de habla. 
que la edad media en las tres zonas es igual, podremos llevar a cabo el análisis sin preocuparnos por la influencia de ésta. La hipótesis planteada es:

- $\mathrm{H}_{\mathrm{o}}: \mu 1=\mu 2=\mu 3$ (La edad media es igual en tres zonas).

- $\mathrm{H}_{\mathrm{i}}$ : No todas las $\mu \mathrm{g}$ son iguales.

La hipótesis nula $\left(\mathrm{H}_{\mathrm{o}}\right)$ no se acepta cuando el $p$-value es inferior a 0,05 y tiene un nivel de confianza del $95 \%$. En caso contrario, no se puede rechazar.

\subsection{Prueba de hipótesis para proporciones}

La prueba de hipótesis para proporciones fue empleada en esta investigación para establecer si existen diferencias significativas en la selección de usted en función de la variable lugar de residencia del informante. Las hipótesis planteadas son:

- $\mathrm{H}_{\mathrm{o}}: p_{1}=p_{2}=p_{3}$ (Las proporciones son iguales en tres zonas).

- $\mathrm{H}_{\mathrm{i}}$ : No todas las $p_{\mathrm{g}}$ son iguales.

La hipótesis nula se rechaza cuando el valor de la significancia asintótica (Asymp. Sig.) es menor que 0,05 , permitiendo afirmar que existen diferencias significativas entre las tres zonas analizadas. De esta manera, se pretendió observar, por un lado, en qué zona se usaría más el usted y, por otro, si habría diferencia acerca de la evaluación de vos y de tú en función de la zona.

\section{AnÁlisis}

De acuerdo con el tamaño de muestra óptimo para distribuciones multinomiales, el tamaño de muestra $n$ estimado fue de 765 personas y, aplicando encuestas a 4.207 personas, se consiguieron 1.490 cuestionarios válidos. Con ello, se obtuvo una cantidad suficiente de informantes para un análisis cuantitativo corroborado estadísticamente. Véase la Tabla 1 para conocer los detalles de los informantes:

Tabla 1: Distribución de informantes

\begin{tabular}{|l|l|r|r|r|r|}
\hline \multirow{2}{*}{$\begin{array}{c}\text { Lugar de vivienda } \\
\text { del informante }\end{array}$} & \multirow{2}{*}{ Sexo } & \multicolumn{3}{|c|}{ Estrato socioeconómico } & \multirow{2}{*}{ Total } \\
\cline { 3 - 6 } & & 1 & 2 & 3 & \\
\hline \multirow{2}{*}{ Área metropolitana } & Femenino & 45 & 256 & 188 & 489 \\
\cline { 2 - 6 } & Masculino & 42 & 187 & 164 & 393 \\
\hline Carmen del Viboral & Femenino & 2 & 68 & 106 & 176 \\
\cline { 2 - 6 } & Masculino & 2 & 25 & 45 & 72 \\
\hline \multirow{2}{*}{ Andes } & Femenino & 32 & 146 & 37 & 215 \\
\cline { 2 - 6 } & Masculino & 15 & 94 & 36 & 145 \\
\hline Total & & $\mathbf{1 3 8}$ & $\mathbf{7 7 6}$ & $\mathbf{5 7 6}$ & $\mathbf{1 4 9 0}$ \\
\hline
\end{tabular}


La prueba del análisis de varianza con un solo factor (ANOVA) nos indica que la edad media en las tres zonas es estadísticamente uniforme (Valor P: 0,804), es decir, la diferencia de la edad en función de la zona no afecta nuestro análisis. Esto nos permite realizar un análisis riguroso sólo con la variable lugar de residencia del informante. Véase la Tabla 2:

Tabla 2: La edad media en tres zonas

\begin{tabular}{|l|c|c|c|c|}
\hline \multirow{2}{*}{ Edad de los informantes } & \multicolumn{3}{|c|}{ Lugar de vivienda } & \multirow{2}{*}{ General } \\
\cline { 2 - 5 } & $\begin{array}{c}\text { Área } \\
\text { Metropolitana }\end{array}$ & $\begin{array}{c}\text { Carmen de } \\
\text { Viboral }\end{array}$ & Andes & 13 \\
\hline Edad mínima & 14 & 14 & 13 & 34 \\
\hline Edad máxima & 34 & 32 & 34 & 19 \\
\hline Edad media & 20 & 16 & 17 & 19 \\
\hline
\end{tabular}

\subsection{La frecuencia del tuteo en tres zonas antioqueñas}

Tabla 3: La frecuencia del $t u ́$

\begin{tabular}{|c|c|c|c|c|}
\hline \multirow{3}{*}{ Preguntas } & \multicolumn{3}{|c|}{ Lugar de vivienda del informante } & \multirow{2}{*}{ Igualdad de proporciones } \\
\hline & Area metropolitana & Carmen de Viboral & Andes & \\
\hline & Tú & Tú & Tú & Sig. \\
\hline 1 & $30.30 \%$ & $24.20 \%$ & $22.80 \%$ & $0,011^{*}$ \\
\hline 2 & $26.10 \%$ & $20.60 \%$ & $18.90 \%$ & $0,013 *$ \\
\hline 3 & $20.90 \%$ & $17.80 \%$ & $17.60 \%$ & 0.313 \\
\hline 4 & $26.20 \%$ & $18.60 \%$ & $17.30 \%$ & $0,001 *$ \\
\hline 5 & $20.90 \%$ & $17.50 \%$ & $16.10 \%$ & 0.113 \\
\hline 6 & $24.30 \%$ & $18.60 \%$ & $17.70 \%$ & $0,018^{*}$ \\
\hline 7 & $25.60 \%$ & $16.90 \%$ & $17.80 \%$ & $0,001 *$ \\
\hline 8 & $25.10 \%$ & $15.90 \%$ & $18.80 \%$ & $0,002 *$ \\
\hline 9 & $41.20 \%$ & $31.60 \%$ & $31.00 \%$ & $0,001^{*}$ \\
\hline 10 & $55.40 \%$ & $39.10 \%$ & $36.20 \%$ & $0,000^{*}$ \\
\hline 11 & $38.90 \%$ & $30.90 \%$ & $29.20 \%$ & $0,002^{*}$ \\
\hline 12 & $55.40 \%$ & $39.10 \%$ & $36.20 \%$ & $0,000^{*}$ \\
\hline 13 & $55.50 \%$ & $48.60 \%$ & $45.30 \%$ & $0,002 *$ \\
\hline 14 & $45.60 \%$ & $35.00 \%$ & $33.60 \%$ & $0,000^{*}$ \\
\hline 15 & $43.90 \%$ & $34.40 \%$ & $31.10 \%$ & $0,000^{*}$ \\
\hline 16 & $48.10 \%$ & $38.50 \%$ & $37.60 \%$ & $0,001 *$ \\
\hline 17 & $49.10 \%$ & $40.70 \%$ & $38.40 \%$ & $0,001 *$ \\
\hline 18 & $20.10 \%$ & $19.40 \%$ & $17.80 \%$ & 0.646 \\
\hline 19 & $24.80 \%$ & $21.80 \%$ & $21.90 \%$ & 0.418 \\
\hline 20 & $17.10 \%$ & $16.10 \%$ & $14.50 \%$ & 0.527 \\
\hline 21 & $20.40 \%$ & $18.20 \%$ & $18.20 \%$ & 0.580 \\
\hline 22 & $11.40 \%$ & $10.10 \%$ & $7.50 \%$ & 0.125 \\
\hline 23 & $13.60 \%$ & $16.90 \%$ & $11.40 \%$ & 0.150 \\
\hline 24 & $11.60 \%$ & $10.90 \%$ & $7.80 \%$ & 0.136 \\
\hline
\end{tabular}




\begin{tabular}{|l|c|c|c|c|}
\hline 25 & $12.70 \%$ & $17.30 \%$ & $6.70 \%$ & $0,000^{*}$ \\
\hline 26 & $48.00 \%$ & $44.40 \%$ & $28.60 \%$ & $0,000^{*}$ \\
\hline 27 & $47.00 \%$ & $42.30 \%$ & $28.80 \%$ & $0,000^{*}$ \\
\hline 28 & $20.10 \%$ & $18.50 \%$ & $17.60 \%$ & 0.575 \\
\hline 29 & $22.30 \%$ & $20.60 \%$ & $15.60 \%$ & $0,027 *$ \\
\hline 30 & $18.40 \%$ & $15.30 \%$ & $12.80 \%$ & $0,047^{*}$ \\
\hline 32 & $18.00 \%$ & $16.20 \%$ & $12.80 \%$ & 0.082 \\
\hline 33 & $11.00 \%$ & $8.90 \%$ & $9.20 \%$ & 0.481 \\
\hline 34 & $11.10 \%$ & $9.30 \%$ & $9.20 \%$ & 0.491 \\
\hline 35 & $10.30 \%$ & $9.70 \%$ & $9.50 \%$ & 0.887 \\
\hline 36 & $10.10 \%$ & $10.90 \%$ & $7.50 \%$ & 0.286 \\
\hline 37 & $11.90 \%$ & $12.90 \%$ & $10.60 \%$ & 0.683 \\
\hline 38 & $13.40 \%$ & $16.90 \%$ & $12.00 \%$ & 0.208 \\
\hline 39 & $11.70 \%$ & $10.10 \%$ & $9.40 \%$ & 0.470 \\
\hline 40 & $11.70 \%$ & $10.10 \%$ & $9.40 \%$ & 0.470 \\
\hline 41 & $9.20 \%$ & $10.10 \%$ & $8.10 \%$ & 0.687 \\
\hline
\end{tabular}

Según los resultados que aparecen en la Tabla $3^{14}$, la estadística muestra que, en la gran mayoría de las relaciones interpersonales solidarias, por ejemplo, entre miembros de la misma familia o amigos, en Medellín (zona urbana) se emplea más el tú que en Carmen de Viboral (zona semirural) y, asimismo, en este último municipio se emplea con mayor frecuencia el tú que en Andes (zona rural). En resumen, en la zona urbana se emplea más el tú que en la zona semirural, y en esta última se emplea más que en la zona rural.

Como se ha descrito en páginas anteriores, a finales del siglo XV, en la época en que los peninsulares llegaron al Nuevo Mundo, la política peninsular exhortaba el uso de vuestra merced y tú y, asimismo, desvalorizaba el vos en América por la doble dimensión problemática del voseo antes mencionada. De esta manera, en Ciudad de México o en Lima donde había virreinatos -o sus alrededores-, más concretamente, donde existía un fácil acceso a la política o la cultura peninsular, rápidamente se siguió este cambio lingüístico. Contrariamente, donde no había esta facilidad geográfica, no se popularizó el cambio. Así, en algunas regiones hispanoamericanas desapareció el voseo y el tuteo fue ganando terreno (Cisneros 1996: 40; Fontanella 1976: 269; Hummel 2010b: 295-296, 2010c: 395; Montes 1967: 22-23; Páez 1981: 76, 98; M. Steffen 2010: 434; Torrejón 2010: 418, 421; Vázquez y Orozco 2010: 249-252, 256; von Büchau 2006: 134). Con base en lo descrito, desde una visión diacrónica a largo plazo, se puede afirmar que el tú en América y también en el departamento colombiano de Antioquia se considera más bien como un trato no propio de esta región, o lo que es lo mismo, es una nueva forma de tratamiento comparado con el vos. Por otro lado, como se ha mostrado anteriormente, en algunas regiones hispanohablantes el tuteo suele predominar en las zonas urbanas y, en cambio, el voseo prevalece en las zonas rurales (Hummel 2010a: 104, 2010b: 311, 2010c: 377, 384-385, 2010d: 401-402; Moser 2010: 278, 282-283; Torrejón 2010: 420-422; Vázquez y Orozco 2010: 257).

14 En la última columna se presentan valores de la significancia asintótica y los que son estadísticamente significativos (menores a 0,05$)$ se marcan con un asterisco $(*)$. 
Teniendo en cuenta lo descrito en este apartado, y el hecho de que las zonas urbanas tienden a propiciar y acoger innovaciones lingüísticas, mientras que las rurales suelen ser conservadoras en este aspecto (Moreno 1992: 350), se puede afirmar que el tuteo en Hispanoamérica, en general, así como en la región estudiada en el presente artículo, en particular, empezó a difundirse a partir de las zonas urbanas (Hummel 2010c: 395). Tal y como se muestra en los resultados de la Tabla 3, es claro el predominio del tuteo en la zona urbana, y del voseo en las zonas rurales en el departamento de Antioquia.

Vale la pena apuntar que, de acuerdo con la Tabla 3, la estadística no muestra diferencias significativas sobre la selección pronominal del tú en función de la zona en la gran mayoría de las relaciones interpersonales no solidarias, por ejemplo, al tratar a desconocidos, profesores no amigos, médicos o sacerdotes. Esto quiere decir que el tuteo en estas relaciones interpersonales no solidarias se utiliza uniforme y escasamente en las tres zonas, esto es, ni siquiera en la zona urbana (Medellín) apenas se emplea el tuteo al referirse a estos interlocutores que ejercen poder o con quienes media una distancia social.

\subsection{La evaluación sobre el tú frente al vos en tres zonas antioqueñas}

Tabla 4: Evaluación positiva del tú frente al vos

\begin{tabular}{|c|c|c|c|}
\hline \multicolumn{3}{|c|}{ Lugar de vivienda } & \multirow{2}{*}{ Sig. Asintótica } \\
\cline { 1 - 3 } Área Metropolitana & Carmen de Viboral & Andes & \\
\hline $75.03 \%$ & $65.69 \%$ & $65.29 \%$ & $0,001 *$ \\
\hline
\end{tabular}

Los resultados de la Tabla 4 nos muestran que más de la mitad de los informantes respondieron "Sí” a la pregunta: “¿Usted considera que el uso del tú (por ejemplo, “tú eres") suena más refinado que el uso del vos (por ejemplo, "vos sos")?". Vale decir, los informantes confirmaron que el tuteo sería más refinado que el voseo, lo cual se ha observado en distintas regiones hispanohablantes (Hummel 2010a: 104, 2010b: 311, 2010c: 377, 384-385, 2010d: 401-402; Moser 2010: 278, 282-283; Torrejón 2010: 420-422; Vázquez y Orozco 2010: 257).

Nótese que la evaluación positiva sobre el tuteo frente al voseo, según la estadística, es mayor en la zona urbana que en las zonas rurales, lo cual puede estar influyendo en la diferencia de frecuencia de uso del tuteo en función de las zonas estudiadas. Más concretamente, los habitantes que gozan la vida urbana, considerada un tanto refinada, desean acercarse a lo refinado y, por ende, usarían más el tú, lo cual perece no interesar tanto a los habitantes de las zonas rurales.

\subsection{Matiz feminizante del tuteo en tres zonas antioqueñas}

Tabla 5: Matiz feminizante del tú antioqueño

\begin{tabular}{|c|c|c|c|}
\hline \multicolumn{3}{|c|}{ Lugar de vivienda } & \multirow{2}{*}{ Sig. Asintótica } \\
\cline { 1 - 3 } Área Metropolitana & Carmen de Viboral & Andes & \\
\hline $54.22 \%$ & $56.90 \%$ & $45.45 \%$ & $0,009 *$ \\
\hline
\end{tabular}


Según la Tabla 5, la mitad de los informantes respondieron "Sí" a la pregunta: “¿Usted considera que el uso del tú (por ejemplo, "tú eres”) entre hombre y hombre puede sonar feminizante (afeminado)?"; a saber, afirmaron que el tuteo entre interlocutores masculinos conllevaría un matiz feminizante, lo cual se ha observado en algunas regiones hispanohablantes (Páez 1981: 105) y en la ciudad de Medellín (Jang 2010: 87). A este respecto, cabe señalar que esta tendencia, tal y como muestra el análisis estadístico, es más pronunciada en la zona urbana o semirural que en la zona rural. Este hecho puede deberse a que el tuteo es muy escaso en la zona rural y, por ello, no se ha generalizado tan claramente este matiz.

\section{Consideraciones Finales}

En los estudios anteriores acerca del uso pronominal medellinense se ha afirmado la vitalidad del voseo en Medellín (Jang 2010, 2012, 2013). En Jang (2013: 77-78) se ha señalado que tal vitalidad puede deberse, por un lado, a que el voseo medellinense forma parte de la identidad social de los habitantes de esta ciudad y, por otro lado, a que el tuteo se ha generalizado hasta cierto punto en comparación con décadas y siglos anteriores.

En el estudio de Jang (2012) sobre la selección pronominal en la población medellinense, con base en la literatura existente sobre el tema (Montes 1967; Moreno 1992: 350; Páez 1981), se partía de la hipótesis de que en la mayoría de las relaciones interpersonales los informantes de origen urbano cuyos padres son del mismo origen emplearían el tú más frecuentemente que el vos, en comparación con los informantes de origen rural cuyos padres son del mismo origen. Sin embargo, esta hipótesis no se corroboró, esto es, no se observaron diferencias estadísticamente significativas sobre el predominio del tuteo o el voseo en función del origen urbano/rural, excepto en algunos pocos casos, especialmente en la relación novio/novia.

No obstante, en el presente análisis basado en un trabajo de campo realizado en tres municipios antioqueños de Colombia, la estadística mostró una clara diferencia en la frecuencia de tú o de vos en función del lugar de residencia de los informantes, a saber, en la gran mayoría de relaciones interpersonales solidarias, por ejemplo, entre familiares o amigos, en Medellín (zona urbana) se usa más el tú que en Carmen de Viboral (zona semirural) y, asimismo, en este último municipio se emplea más el tú que en Andes (zona rural). A partir de este resultado, se puede afirmar que en el departamento de Antioquia está ocurriendo lo mismo que se observó en otras regiones hispanohablantes, esto es, el paso del vos al tú desde la zona urbana a la rural por las razones históricas, geográficas y socioeconómicas mencionadas en los apartados anteriores.

En lo que se refiere a la diferencia entre el tuteo y el voseo en Medellín a mediados del siglo $\mathrm{XX}$, cabe referirse al siguiente fragmento observado en una novela autobiográfica de un escritor medellinense: "En Antioquia se habla de vos, y sólo se le da el tú a Dios, o a la novia. Muy sofisticado" (Vallejo 2002: 38). De esta cita se infiere que hace unas décadas en la ciudad de Medellín o en el departamento de Antioquia el tuteo era muy escaso. Igualmente, se deduce que el tuteo se ha venido expandiendo gradualmente en la zona estudiada, ya que, como muestra la Tabla 3, no es un trato exclusivo para la novia, aunque sigue siendo escaso su uso en comparación con el voseo. 
En cierto sentido, como apunta Murillo (2003: 135) para el caso de Costa Rica, y como señalan Álvarez y Carrera (2006: 124-125) para Venezuela, el tratamiento de tú puede estar difundiéndose paulatinamente en la zona estudiada a través de los medios de comunicación, y su uso se ha generalizado en comparación con décadas y siglos anteriores (Jang 2013), aunque no alcance al nivel del voseo (Jang 2010, 2012). Montes (1967) afirmaba que con el tiempo podría desaparecer el voseo de todo el país, no obstante, debido al matiz feminizante del tuteo y al hecho de que se considere el voseo como expresión de la identidad antioqueña (Jang 2013), podemos reafirmar que, como apunta Cisneros (1998: 90), para el mundo voseante en general, el voseo antioqueño no va a desaparecer al menos a corto plazo, lo cual ha sido señalado igualmente en los trabajos de Jang $(2009,2013)$.

\section{OBRAS CITADAS}

Álvarez, Alexandra y Micaela Carrera. 2006. "El usted de solidaridad en el habla de Mérida". En Schrader-Kniffki, Martina (Ed.), La cortesía en el mundo hispánico. Nuevos contextos, nuevos enfoques metodológicos. Frankfurt: Vervuert Iberoamericana. 117-130.

Blas, José. 1994-1995. "Tú y usted: Dos pronombres de cortesía en el español actual. Datos de una comunidad peninsular”. Estudios de Lingüística 10: 21-44.

1995. "Los pronombres de tratamiento y la cortesía". Revista de Estudios Hispánicos 22: 439-466.

2003. "Perspectivas (socio)lingüísticas complementarias en el estudio de la variación y el cambio lingüístico en español”. Estudios de Sociolingüística 4.2: 653-692.

2005. "Los grados de la cortesía verbal: Reflexiones en torno a algunas estrategias comunicativas y recursos lingüísticos en el español peninsular contemporáneo". Revista Internacional de Lingüistica Iberoamericana 3.1: 9-29.

Bolívar, Adriana. 2001. "El insulto como estrategia en el diálogo político venezolano". Oralia 4: 47-73.

Boretti, Susana y Eliabeth Rigatuso. 2004. "La investigación de la cortesía en el español de la Argentina. Estado de la cuestión”. En Bravo, Diana y Antonio Briz (Eds.), Pragmática sociocultural: Estudios sobre el discurso de cortesía en español. Barcelona: Ariel Lingüística. 137-167.

Braun, Friederike. 1988. Terms of address. Problems of usage in various languages and cultures. Berlin: Mouton de Gruyter.

Bravo, Diana. 2001. "Sobre la cortesía lingüística, estratégica y conversacional en español". Oralia 4: 299-314.

Brown, Roger y Albert Gilman. 1960. "The pronouns of power and solidarity”. En Sebeok, Thomas (Ed.), Style in language. Cambridge-Massachusetts: MIT Press. 253-276.

Brown, Penelope y Stephen Levinson. 1987. Politeness. Some universals in language usage. Cambridge: Cambridge University Press.

Carrera, Micaela y Alexandra Álvarez. 2004. "Tratamientos y cortesía en la elaboración de fuentes documentales de la etapa fundacional de la provincia de Mérida (Venezuela)". En Bravo, Diana y Antonio Briz (Eds.), Pragmática sociocultural: Estudios sobre el discurso de cortesía en español. Barcelona: Ariel Lingüística. 227-243.

Cisneros, Mireya. 1996. “Aspectos histórico-pragmáticos del voseo”. Thesaurus 51.1: 27-43. 1998. "Hacia un estudio del voseo. Aspectos históricos, pragmáticos y morfológicos de los tratamientos de segunda persona singular". Litterae 7: 76-94.

Fontanella, María Beatriz. 1970. "La evolución de los pronombres de tratamiento en el español bonaerense". Thesaurus 25.1: 12-22. 
1976. "Analogía y confluencia paradigmática en formas verbales de voseo". Thesaurus 31.2: $249-272$.

1985. "La evolución del voseo bonaerense en el siglo XIX". Estudios Filológicos 20: 9-24.

Guerra, Nuria. 2008. "El discurso conflictivo en interacciones coloquiales en inglés y español. El uso de las fórmulas de tratamiento". En Briz, Antonio et al. (Eds.), Cortesía y conversación: De lo escrito a lo oral. III Coloquio Internacional del Programa EDICE. Valencia-Estocolmo: Universidad de Valencia-Programa EDICE. 224-239.

Hummel, Martin. 2010a. "Reflexiones metodológicas y teóricas sobre el estudio de las formas de tratamiento en el mundo hispanohablante, a partir de una investigación en Santiago de Chile". En Hummel, Martin, Betina Kluge y María Eugenia Vázquez (Eds.), Formas y fórmulas de tratamiento en el mundo hispánico. México D.F.: Colegio de México. 101-162.

2010b. "El estudio de las formas de tratamiento en las Antillas hispanohablantes". En Hummel, Martin, Betina Kluge y María Eugenia Vázquez (Eds.), Formas y fórmulas de tratamiento en el mundo hispánico. México D.F.: Colegio de México. 293-323.

2010c. "El estudio de las formas de tratamiento en Perú". En Hummel, Martin, Betina Kluge y María Eugenia Vázquez (Eds.), Formas y fórmulas de tratamiento en el mundo hispánico. México D.F.: Colegio de México. 375-398.

2010d. "Las formas y fórmulas de tratamiento en Bolivia". En Hummel, Martin, Betina Kluge y María Eugenia Vázquez (Eds.), Formas y fórmulas de tratamiento en el mundo hispánico. México D.F.: Colegio de México. 399-411.

Iglesias, Silvia. 2001. "Los estudios de la cortesía en el mundo hispánico. Estado de la cuestión". Oralia 4: 245-298.

Jang, Ji Son. 2009. Fórmulas de tratamiento pronominales en los jóvenes universitarios de Medellín: Una aproximación socio-pragmática. Disertación doctoral. Osaka: Kansai Gaidai University.

2010. "Fórmulas de tratamiento pronominales en los jóvenes universitarios de Medellín (Colombia) desde la óptica socio-pragmática: Estrato socioeconómico y sexo". Íkala 15.26: 43-116.

. 2012. "Correlaciones entre la selección pronominal, el origen urbano/rural y la edad: El caso de jóvenes universitarios de Medellín (Colombia)". Íkala 17.2: 145-166.

2013. "Voseo medellinense como expresión de identidad paisa". Íkala 18.1: 61-81.

Kluge, Betina. 2005. "Las fórmulas de tratamiento en un corpus chileno". En Noll, Volker, Klaus Zimmermann e Ingrid Neumann-Holzschuh (Eds.), El español en América: Aspectos teóricos, particularidades, contactos. Frankfurt: Vervuert Iberoamericana. 169-188.

Medina, Javier. 2010. "El tuteo presidencial: ¿Descortesía o cambio de "talante”?”. En Orletti, Franca y Laura Mariottini (Eds.), (Des)cortesía en español. Espacios teóricos y metodológicos para su estudio. Roma-Estocolmo: Università degli Studi Roma Tre-Programa Edice. 209-220.

Móccero, María Leticia. 2003. "Las selecciones pronominales como estrategias de cortesía". En Bravo, Diana (Ed.), Actas del Primer Coloquio del Programa EDICE: "La perspectiva no etnocentrista de la cortesía: Identidad sociocultural de las comunidades hispanohablantes". Estocolmo: Universidad de Estocolmo. 346-354.

Molina, Isabel. 2002. "Evolución de las fórmulas de tratamiento en la juventud madrileña a lo largo del siglo XX: Un estudio en tiempo real”. En Rodríguez, Félix (Coord.), El lenguaje de los jóvenes. Barcelona: Ariel. 97-121.

Montes, José. 1967. "Sobre el voseo en Colombia". Thesaurus 22.1: 21-44.

Moreno, Francisco. 1986. "Sociolingüística de los tratamientos. Estudio sobre una comunidad rural". Anuario de Letras 24: 87-120.

1992. "Norma y prestigio en el español de América. Apuntes para una planificación de 
la lengua española”. Revista de Filología Española 72.3/4: 345-359.

Moser, Karolin. 2010. "Las formas de tratamiento verbales-pronominales en Guatemala, El Salvador, Panamá (y Costa Rica): Hacia una nueva sistematización en la periferia centroamericana". En Hummel, Martin, Betina Kluge y María Eugenia Vázquez (Eds.), Formas y fórmulas de tratamiento en el mundo hispánico. México D.F.: Colegio de México. 271-291.

Murillo, Jorge. 2003. "La cortesía verbal en situaciones de habla en Costa Rica: Hacia la comprensión de la imagen social en su contexto sociocultural". En Bravo, Diana (Ed.), Actas del Primer Coloquio del Programa EDICE: "La perspectiva no etnocentrista de la cortesía: Identidad sociocultural de las comunidades hispanohablantes". Estocolmo: Universidad de Estocolmo.128-142.

Navarro, Rosario. 2004. "Formas de cortesía en la "Segunda Celestina"”. En Bravo, Diana y Antonio Briz (Eds.), Pragmática sociocultural: Estudios sobre el discurso de cortesía en español. Barcelona: Ariel Lingüística. 213-225.

Páez, Iraset. 1981. Historia y geografía hispanoamericana del voseo. Caracas: La Casa de Bello.

Pedroviejo, Juan. 2004. "Formas de tratamientos en dos obras de teatro del siglo XX: Historia de una escalera y Bajarse al moro". En Bravo, Diana y Antonio Briz (Eds.), Pragmática sociocultural: Estudios sobre el discurso de cortesía en español. Barcelona: Ariel Lingüística. 245-262.

Placencia, María Elena. 2010. "El estudio de formas de tratamiento en Colombia y Ecuador". En Hummel, Martin, Betina Kluge y María Eugenia Vázquez (Eds.), Formas y fórmulas de tratamiento en el mundo hispánico. México D.F.: Colegio de México. 341-373.

Serrano, María José. 2000. "La producción de la cortesía verbal y la deixis socio-comunicativa”. Oralia 3: 199-219.

Steffen, Joachim. 2010. "El tratamiento en Uruguay”. En Hummel, Martin, Betina Kluge y María Eugenia Vázquez (Eds.), Formas y fórmulas de tratamiento en el mundo hispánico. México D.F.: Colegio de México. 449-464.

Steffen, Martina. 2010. "El tratamiento en Paraguay". En Hummel, Martin, Betina Kluge y María Eugenia Vázquez (Eds.), Formas y fórmulas de tratamiento en el mundo hispánico. México D.F.: Colegio de México. 429-448.

Thompson, Steven. 1987. "Sample size for estimating proportions". The American Statistician 41.1: 42-46.

Torrejón, Alfredo. 2010. "El voseo en Chile: Una aproximación diacrónica”. En Hummel, Martin, Betina Kluge y María Eugenia Vázquez (Eds.), Formas y fórmulas de tratamiento en el mundo hispánico. México D.F.: Colegio de México. 413-427.

Uber, Diane. 2010. "Formas y fórmulas de trato en situaciones laborales en Santiago de Chile y Buenos Aires”. En Hummel, Martin, Betina Kluge y María Eugenia Vázquez (Eds.), Formas y fórmulas de tratamiento en el mundo hispánico. México D.F.: Colegio de México. 1051-1080.

Vallejo, Fernando. 2002. El río del tiempo. Bogotá: Editorial Alfaguara.

Vázquez, María Eugenia y Leonor Orozco. 2010. "Formas de trato del español en México". En Hummel, Martin, Betina Kluge y María Eugenia Vázquez (Eds.), Formas y fórmulas de tratamiento en el mundo hispánico. México D.F.: Colegio de México. 247-269.

Von Büchau, Michael. 2006. "Las formas de tratamiento en el español cubano del siglo XIX". En Schrader-Kniffki, Martina (Coord.), La cortesía en el mundo hispánico. Nuevos contextos, nuevos enfoques metodológicos. Frankfurt: Vervuert Iberoamericana. 131-138. 
